\title{
Sequential Generation of Projected Entangled-Pair States
}

\author{
Zhi-Yuan Wei®, ${ }^{*}$ Daniel Malz@, ${ }^{*}$ and J. Ignacio Cirac® \\ Max-Planck-Institut für Quantenoptik, Hans-Kopfermann-Straße 1, D-85748 Garching, Germany \\ and Munich Center for Quantum Science and Technology (MCQST), Schellingstraße 4, D-80799 München, Germany
}

(Received 10 August 2021; revised 30 October 2021; accepted 30 November 2021; published 7 January 2022)

\begin{abstract}
We introduce plaquette projected entangled-pair states, a class of states in a lattice that can be generated by applying sequential unitaries acting on plaquettes of overlapping regions. They satisfy area-law entanglement, possess long-range correlations, and naturally generalize other relevant classes of tensor network states. We identify a subclass that can be more efficiently prepared in a radial fashion and that contains the family of isometric tensor network states [M. P. Zaletel and F. Pollmann, Phys. Rev. Lett. 124, 037201 (2020)]. We also show how this subclass can be efficiently prepared using an array of photon sources.
\end{abstract}

DOI: 10.1103/PhysRevLett.128.010607

Tensor network states play a fundamental role both in quantum information processing and many-body physics, as they are natural representations of states with area-law entanglement [1-3]. In one dimension, matrix-product states (MPS) [4-6] efficiently approximate the ground state of gapped [7] and critical Hamiltonians [8]. Their higherdimensional generalizations, projected entangled-pair states (PEPS) [9], also play an important role in many-body physics. Apart from providing efficient approximations in different scenarios, they embrace many paradigmatic states of condensed matter physics, including topological states like the toric code [10,11] and string-net states [12-14], or resonating valence bound states [10]. They also contain elements that are relevant in the context of quantum metrology [15], like the W [16] or GHZ states [17], or in quantum computing, like the cluster [18], graph [19-21], and hypergraph states $[22,23]$. Thus, the efficient preparation of such states would have an important impact on the study of many-body systems and quantum information.

One can generate MPS by sequentially applying local unitaries $[24,25]$, which provides a way to deterministically prepare entangled states on quantum computers [26,27] or in photonic systems [25,28-36], with a generation time (circuit depth) that scales linearly with the system size $n$ (number of qudits) as $O(n)$. Moreover, sequential MPS generation is an essential component in numerous theoretical frameworks [27,37-45].

Efficient generation of PEPS is, however, much more difficult. Even in two dimensions, it is believed that most

Published by the American Physical Society under the terms of the Creative Commons Attribution 4.0 International license. Further distribution of this work must maintain attribution to the author(s) and the published article's title, journal citation, and DOI. Open access publication funded by the Max Planck Society. states will require a preparation time that increases exponentially with the system size $[10,46]$. Nevertheless, most of the paradigmatic examples mentioned above can also be efficiently prepared in higher dimensions, and experimental efforts have already started $[47,48]$. This calls for efforts to identify, classify, and extend subclasses of PEPS that allow for efficient preparation, ideally together with an explicit algorithm to do so. In this vein, there are two subclasses of PEPS in two dimensions that stand out: (i) sequentially generated states (SGS) [49], and (ii) PEPS generated by photon feedback (F-PEPS) [50]. Interestingly, both of these classes can be obtained from a product state by a sequential quantum circuit.

In this Letter, we introduce plaquette PEPS (P-PEPS), which are defined by sequentially applying unitaries to plaquettes of qudits initially in a product state. P-PEPS can straightforwardly be expressed as PEPS and naturally encompass SGS and F-PEPS. We focus on a particular radial plaquette ordering, which leads to a subclass we call radial plaquette PEPS (RP-PEPS). This class allows certain local observables to be computed efficiently and has SGS and isometric tensor network states (isoTNS) [51] as proper subclasses. Thus, our construction provides a quantum circuit to prepare isoTNS, which is a class that has been shown to include graph states and hypergraph states of local connectivity, and all string-net states [52]. While for a $q$-dimensional lattice of $N=n_{1} \times \ldots \times n_{q}$ sites, in the worst case, P-PEPS require a circuit depth scaling with the total number of sites, RP-PEPS can be prepared particularly efficiently, with the circuit depth $T_{\mathrm{RP}}$ scaling as the side length of the lattice

$$
T_{\mathrm{RP}}=O\left(\max _{i} n_{i}\right)
$$

We also show that an array of coupled quantum sources, each comprising an ancilla-emitter pair, can naturally 
produce RP-PEPS of flying qubits or qudits with the same efficient scaling, and prepare F-PEPS with a circuit depth $O(N)$. This includes a wide variety of highdimensional states that have been proposed for sequential photon generation [20,35,50,53-62]. Overall, P-PEPS (RPPEPS) and their generation protocols apply to photonic systems, and to platforms with matter qubits or qudits like superconducting circuits [63], trapped ions [64], or Rydberg atoms [65], where local interactions can be engineered with high precision.

Plaquette PEPS.-For concreteness, we restrict our attention to a two-dimensional lattice of qudits of size $N=n \times m$, and the high-dimensional generalization will be discussed in the Supplemental Material (SM) [66].

We define P-PEPS with periodic boundary conditions as the states generated from the product state $|0\rangle^{\otimes N}$ through sequential application of unitaries to plaquettes of size $L_{p} \times L_{p}\left(L_{p} \ll m, n\right)$ [cf. Fig. 1(a)]

$$
\left|\psi_{\mathrm{p}}\right\rangle=\prod_{\mu=1}^{N} \hat{U}_{\vec{v}_{\mu}}|0\rangle^{\otimes N},
$$

where $\vec{v}_{\mu}=\left(i_{\mu}, j_{\mu}\right)$ and the unitary $\hat{U}_{\vec{v}_{\mu}}$ acts on qudits in the square spanning from $\left(i_{\mu}, j_{\mu}\right)$ to $\left(i_{\mu}+L_{p}-1, j_{\mu}+L_{p}-1\right)$, and we identify the rows $i \pm m \equiv i$ and columns $j \pm n \equiv j$. Here, the choice of plaquette shape reflects the locality of the unitaries. The ordering of the unitaries $\mathcal{P}=$ $\left(\vec{v}_{1}, \vec{v}_{2}, \ldots, \vec{v}_{N}\right)$ fulfils the conditions $\vec{v}_{\mu} \neq \vec{v}_{\nu}$ for $\mu \neq \nu$. We show an example of $\mathcal{P}$ in Fig. 1(b) and call the position of the first unitary $\vec{v}_{1}$ the source point. To define the state with open boundary conditions, we simply omit gates that act across boundaries.

P-PEPS is a subclass of PEPS, which are states defined through a network of tensors with one tensor per lattice site [Fig. 1(c)], whose virtual indices are contracted with their neighbors. In two dimensions,

$$
\left.\left|\Psi_{\mathrm{PEPS}}\right\rangle=\sum_{\{k\}=0}^{d-1} \mathcal{F}_{2 \mathrm{D}}\left(\left\{B_{[i, j]}^{k}\right]_{\text {lurb }}\right\}\right)|\{k\}\rangle,
$$

where $B_{[i, j]}^{k}$ iurb is a rank-five tensor on the site $(i, j)$ that has one physical index $k$ of dimension $d$ and four virtual indices $l, u, r, b$ of bond dimension $D$. The symbol $\mathcal{F}_{2 \mathrm{D}}$ denotes the contraction of all virtual indices. To obtain the PEPS representation of P-PEPS, we decompose the plaquette unitaries into projected entangled-pair operators (PEPO) [3] [cf. Fig. 1(d)]. This allows one to write the whole sequential circuit as a PEPO, which, applied to a product state, yields a PEPS with bond dimension $D \leq O\left(d^{L_{p}^{4}}\right)$ [66].

We are particularly interested in cases where each unitary overlaps with at least one of the earlier ones, such that they create correlations. The state shown in Fig. 1(a) is such an example. Sequential circuits with overlapping unitaries

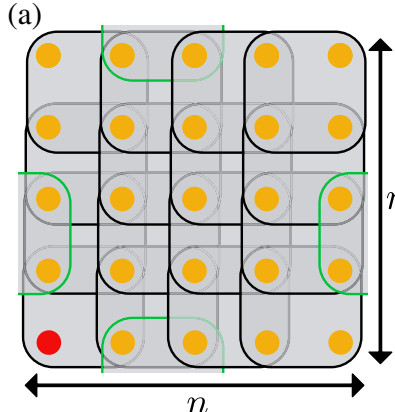

(b)

(c)

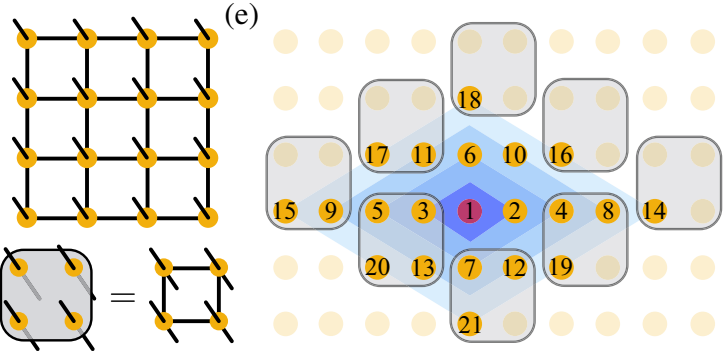

FIG. 1. (a) Plaquette PEPS (P-PEPS) are prepared by sequentially applying plaquette unitaries $\left\{\hat{U}_{\vec{v}_{\mu}}\right\}$ (denoted by the gray squares) of size $L_{p} \times L_{p}\left(L_{p}=2\right.$ here) to a product state. The source point $\vec{v}_{1}$ is marked by a red dot. Periodic and open boundary conditions are distinguished by whether or not the sequence of unitaries contains that act across the boundary (green squares). (b) P-PEPS is determined by the unitaries and their ordering $\mathcal{P}$. Here, we show an example of $\mathcal{P}$ using a directional string and the numbers from 1 to $N$. (c) PEPS are states defined through connected networks of tensors with virtual indices (connected lines) of bond dimension $D$, and physical indices of dimension $d$ (sticking out). (d) The plaquette unitaries can be decomposed into PEPO, which lead to a PEPS representation of P-PEPS. (e) Preparation of a radial plaquette PEPS with $L_{p}=2$. Here, starting from the source point (the red dot), the ordering $\mathcal{P}$ is denoted by the numbers. Such ordering allows one to apply each layer (denoted by the shades of different colors) of unitaries in parallel. The gates in the fifth layer are denoted by gray squares.

efficiently establish correlations between arbitrary locations of the lattice with $O(N)$ unitaries [66]. This should be contrasted with brick wall circuits [69] that take $O\left(N \cdot \max _{i} n_{i}\right)$ unitaries to do so [66]. This implies that P-PEPS offer a more efficient parametrization of states with correlations across the entire system. Moreover, while P-PEPS have area-law entanglement, brick wall circuits that create long-range correlations will, instead, lead to states with volume-law entanglement [70-72].

Radial plaquette PEPS.-Naively, it takes a circuit depth $O(N)$ to create a P-PEPS [cf. Eq. (2)]. However, some orderings $\mathcal{P}$ allow unitaries to be applied in parallel. A simple example is to arrange the unitaries as a brick wall circuit of depth $O\left(L_{p}^{2}\right)$. Here, we define a subclass of P-PEPS, RP-PEPS, where starting from the source point, the positions $\left\{\vec{v}_{\mu}\right\}$ of the unitaries are ordered such that they can be grouped to multiple layers of commuting 
unitaries, and each layer acts on the boundary of the existing gate-acted region. An example with $L_{p}=2$ is illustrated in Fig. 1(e), where the gates are grouped as $[(1),(2,3),(4-7),(8-13),(14-21), \ldots]$ (denoted by shades of different colors). To resolve ambiguities in the plaquette order, we choose preferred directions in which the position of the plaquette moves. In Fig. 1(e), we choose "horizontal first, and positive direction first." The circuit depth of preparing RP-PEPS is asymptotically $T_{\mathrm{RP}} \approx n+L_{p} m$, following the scaling as Eq. (1). Moreover, RP-PEPS allow efficient computation of expectation values of local observables that are geometrically close to the source point or the line that passes through the source point along the preferred direction, and this generically implies that correlation functions in these regions decay exponentially. This is reminiscent of isoTNS [51].

The above definitions straightforwardly generalize to higher dimensional lattices, where plaquettes become highdimensional cubes [66]. While the general circuit depth for P-PEPS again scales with $N$, RP-PEPS obeys Eq. (1).

Relation to other families of PEPS.-By definition, PPEPS can be efficiently prepared, have a PEPS description, and host long-range correlations. Now, we show that PPEPS naturally encompass other families of PEPS that are prepared sequentially (SGS and F-PEPS), as well as isoTNS (we follow the definition in Ref. [51], and see Ref. [73] for a different definition).

SGS [cf. Fig. 2(a)] are defined in terms of linear sequential circuits comprising unitaries $\left\{\hat{V}_{[i, j]}\right\}$ of length $L_{p}$ acting on rows across qudits whose columns have been prepared in MPS [49]
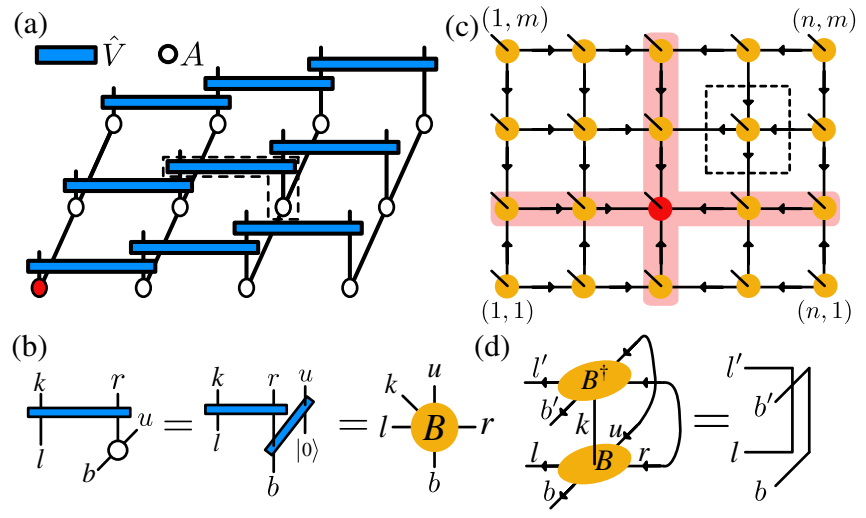

FIG. 2. (a) $2 \mathrm{D}$ SGS are constructed by first preparing multiple columns of MPS $\left\{\left|\psi_{\text {MPS }}^{i^{\prime}}\right\rangle\right\}$ with tensors $\{A\}$, and then coupling neighboring columns of MPS with linear sequential unitaries $\{\hat{V}\}$ of length $L_{p}\left(L_{p}=2\right.$, here). The red dot denotes the source point of the corresponding P-PEPS. (b) The tensor inside the dashed box in (a) can be viewed as two connected two-qudit unitaries and identified as a PEPS tensor. (c) Isometric tensor network states (isoTNS). The red shaded lines (dot) are the orthogonality hypersurfaces (orthogonality center). (d) The isometry condition of the tensor inside the dashed box in (a) and (c).

$$
\left|\psi_{\mathrm{SGS}}\right\rangle=\prod_{i=1}^{n-s} \prod_{j=1}^{m} \hat{V}_{[i, j]} \otimes_{i^{\prime}=1}^{n}\left|\psi_{\mathrm{MPS}}^{i^{\prime}}\right\rangle .
$$

The MPS in each column can be put in canonical form, such that they can be written as linear sequential circuits $[24,49]$. This allows us to identify the tensor of the corresponding PEPS as two overlapping $L_{p}$-qudit unitaries [cf. Fig. 2(b)]. These two unitaries are contained in a $L_{p} \times$ $L_{p}$ plaquette unitary. Thus, each SGS can be written as a RP-PEPS, with the source point at the bottom left of the lattice in the case of Fig. 2(a).

To be precise, let us denote the class of SGS (RP-PEPS) on an $n \times m$ lattice with circuit length (plaquette length) $L_{p}$ as $\operatorname{SGS}_{n \times m}^{L_{p}}\left(\mathrm{RP}_{\mathrm{PEPS}} \mathrm{L}_{n \times m}^{L_{p}}\right)$, we have

$$
\mathrm{SGS}_{n \times m}^{L_{p}} \subset \mathrm{RP}-\mathrm{PEPS} \mathrm{S}_{n \times m}^{L_{p}} .
$$

The tensors of SGS in the bulk satisfy an isometry condition shown in Fig. 2(d), which is the same condition as is obeyed by the tensors in isoTNS [51]. Indeed, as we show in the following, these classes are closely related.

isoTNS are PEPS [Eq. (3)] in which all tensors satisfy isometry conditions that depend on their position in the lattice. Specifically, when all incoming indices of a tensor [denoted by incoming arrows in Fig. 2(c)] and the physical index are contracted with corresponding indices of the complex conjugate of that tensor, the remaining indices yield the identity. For example, the tensor in the dashed box in Fig. 2(c) obeys [cf. Fig. 2(d)]

$$
\sum_{k, u r} B_{[i, j] l u r b}^{k}\left(B_{[i, j] l^{\prime} u r b^{\prime}}^{k}\right)^{*}=\delta_{b b^{\prime}} \delta_{l l^{\prime}} .
$$

The red shaded lines in Fig. 2(c) are called orthogonality hypersurfaces, which only have incoming arrows, and their intersection is the orthogonality center (OC) [51].

One can prepare isoTNS as RP-PEPS, but restricting the unitaries in the bulk to be "L" shaped, as shown in Figs. 3(a1)-3(a6). The required three-qudit unitaries can be written as

$$
\left.\hat{B}_{[i, j]}=\sum_{l u r b, k} B_{[i, j]}^{k}\right]_{l u r b}|k, r, u\rangle\langle l, b, 0|,
$$

where $|k, r, u\rangle \equiv\left|k_{[i, j]}, r_{[i, j+1]}, u_{[i+1, j+1]}\right\rangle$, and the tensor $B_{[i, j]_{l u r b}}^{k}$ automatically satisfies the isometry condition Eq. (6). Thus, each unitary creates an isoTNS site [cf. Fig. 3(b)].

Sequentially applying the gates shown in Figs. 3(a1)-3(a6) gives rise to the tensor contraction pattern shown in Fig. 3(a7), which represents an arbitrary isoTNS with OC in the corner. The generated isoTNS has bond dimension $d$ and physical dimension $d$, except at the right boundary, where two sites of each row are combined to 

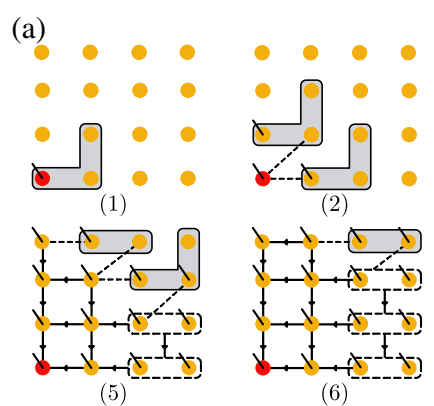

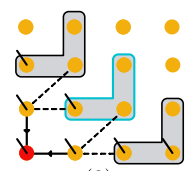

(3)

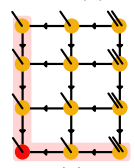

(7)

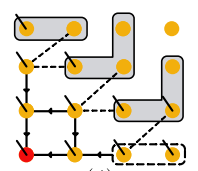

(4)

(b)

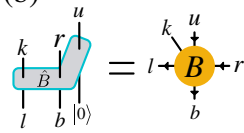

$b$
. (a) The protocol starts from an initial state $|0\rangle^{\otimes N}$, and we sequentially apply three-qudit unitaries that couple neighboring qudits vertically and horizontally following the order in steps 1-6. Each unitary becomes a tensor of the resulting PEPS at the location indicated by the index sticking out. The dashed lines are guiding lines to indicate the connection to previously applied unitary blocks. At the end (4-6), we group the sites of the last two columns (dashed boxes) to satisfy the isometry conditions. This circuit can prepare arbitrary isoTNS [cf. Fig. 2(c)] of the geometry shown in step 7 , with bond dimension $d$, and physical dimension $d$. (b) Each three-qudit unitary can be identified as an isoTNS tensor, which satisfies the isometry condition Eq. (6).

form a site with physical dimension $d^{2}$. Note that arbitrary isoTNS of that geometry with a uniform physical dimension can be embedded in that state by setting the rightmost qudits to zero and treating them as ancillas. Moreover, it is clear from Fig. 3(a) that the circuit depth for preparing isoTNS is $T_{\text {iso }} \approx n+m$. In the SM [66], we show that: (i) by extending the indices of L-shaped unitaries to $2 s+1$ qudits with $s=\left\lceil\log _{d} D\right\rceil$ and changing the source point of the RP-PEPS, isoTNS with arbitrary bond dimension $D$ and with OC in the bulk can be prepared. (ii) This protocol can be generalized to prepare isoTNS of higher dimensions. Therefore, isoTNS on arbitrary lattices (of size $n_{1} \times \ldots \times n_{q}$ ) admit exact representations as sequential quantum circuits, with the circuit depth scaling as

$$
T_{\text {iso }} \approx \sum_{i=1}^{q} n_{i} .
$$

The above observation shows that isoTNS $\subset$ RP-PEPS. A similar relation also holds between F-PEPS [50] and P-PEPS, that F-PEPS $\subset$ P-PEPS. Here, F-PEPS is understood as a generalization to qudits and with arbitrary photon feedback. FPEPS can be viewed as isoTNS on a lattice with different connectivity [50]. If we denote isoTNS ${ }_{n \times m}^{D, d}\left(\mathrm{~F}^{-} \mathrm{PEPS}_{n \times m}^{D, d}\right)$ as the class of isoTNS (F-PEPS) on a $n \times m$ lattice with bond dimension $D$ and physical dimension $d$, we prove in the $\mathrm{SM}$ [66] that isoTNS (F-PEPS) are contained in RP-PEPS (PPEPS) with a slightly larger lattice

$$
\begin{gathered}
\operatorname{isoTNS}_{n \times m}^{D, d} \subset \operatorname{RP}^{-P E P S} \\
\text { F-PEPS }_{n \times m}^{D, d} \subset \operatorname{P}_{(n+2 s) \times(m+2 s)}, \operatorname{PEPS}_{(n+s) \times(m+s)}^{2 s+1} .
\end{gathered}
$$

Having established that both SGS and isoTNS are RPPEPS with L-shaped unitaries, we note that SGS have a further condition on the unitaries, namely that they can be decomposed into two unitaries corresponding to the two arms of the L [see Fig. 2(b)], which indicates that SGS are a subclass of isoTNS. This has direct consequences for the states. While in SGS, local observables can efficiently be calculated anywhere in the lattice, in isoTNS, this requires shifting the OC, which can only be done approximately. Their precise relation is [66]

$$
\mathrm{SGS}_{n \times m}^{L_{p}} \subset \operatorname{isoTNS} d_{n \times m}^{d^{L_{p}\left(L_{p}-1\right)}, d}
$$

Finally, we note that, since the isometry of the PEPS tensors derives directly from the unitarity of the preparation circuit, we further show that RP-PEPS can be expressed as isoTNS on lattices with unusual connectivities [66].

Generating RP-PEPS of flying qubits or qudits.Reference [24] introduces a protocol to prepare arbitrary photonic MPS of bond dimension $D$ and physical dimension $d$ using a photon source comprising a $D$-level ancilla $A_{1}$ and a $d$-level emitter $E_{1}$. The MPS is prepared by repeatedly applying a unitary on the joint ancilla-emitter system, followed by swapping the emitter state into a flying photon, defined in terms of the photon emission isometry $M_{\mathrm{ph}}$

$$
M_{\mathrm{ph}}:|k\rangle_{d} \rightarrow|0\rangle_{d}|k\rangle_{\mathrm{ph}}, \quad k \in(0, \ldots, d-1) .
$$

Now, we extend the above protocol by considering an array of $m$ sequential photon sources coupled to each other as shown in Fig. 4 and show that photonic RP-PEPS can be prepared with this setup.

The protocol is shown in Fig. 4. Here, we assume each ancilla has a dimension $D=d^{L_{p}-1}$, so it can be thought of as $L_{p}-1$ qudits. Starting with the ancillas $\left\{A_{j}\right\}$ and emitters $\left\{E_{j}\right\}$ in their ground state $\left|\varphi_{0}\right\rangle=\left|0_{\left\{A_{j}\right\}}\right\rangle \otimes\left|0_{\left\{E_{j}\right\}}\right\rangle$, in the step to prepare the $(i, j)$-th site of the RP-PEPS, first, we

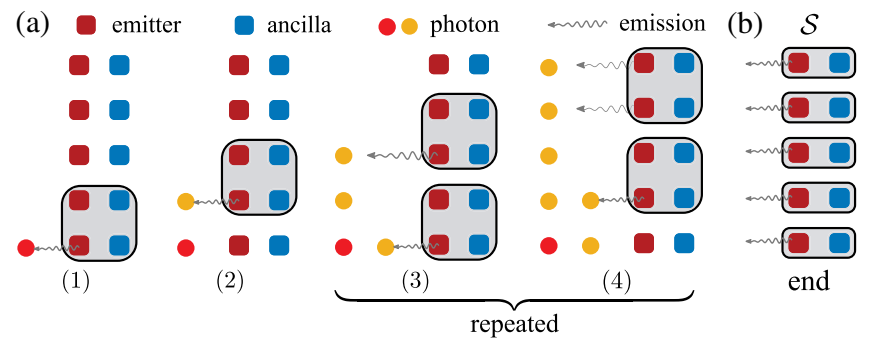

FIG. 4. Generation of photonic RP-PEPS using an array of coupled sequential photon sources. (a) In the preparation of the $[i, j]$-th RP-PEPS site, we apply a unitary $\hat{U}_{[i, j]}$ followed by a photon emission $M_{\mathrm{ph}}^{i}$ of the emitter $E_{j}$. After the initial steps (1) and (2), steps (3) and (4) will be repeated. (b) At the end of the protocol, we swap the excitations on the ancillas to the emitters and then convert them to photons, denoted as $\mathcal{S}$. 
apply a unitary $\hat{U}_{[i, j]}$ that acts on the ancillas $\left\{A_{j}, \ldots\right.$, $\left.A_{j+L_{p}-1}\right\}$ and emitters $\left\{E_{j}, \ldots, E_{j+L_{p}-1}\right\}$ [see Fig. 4(a) for $L_{p}=2$ case]. After the unitary, we trigger the photon emission from the emitter $E_{j}$ (denoted as $M_{\mathrm{ph}}^{j}$ [cf. Eq. (12)]). To disentangle the ancilla from the photons, in the last $L_{p}-1$ steps of the protocol, we sequentially swap the effective $L_{p}-1$ qudits contained in the ancilla into photons [cf. Fig. 4(b)], an operation collectively denoted by $\mathcal{S}$. The final state of the system is $\left|\varphi_{0}\right\rangle\left|\psi_{\mathrm{RP}}\right\rangle_{\mathrm{ph}}$, with the photonic state

$$
\left|\psi_{\mathrm{RP}}\right\rangle_{\mathrm{ph}}=\left\langle\varphi_{0}\left|\mathcal{S} \prod_{i=1}^{n-L_{p}+1} \prod_{j=1}^{m-L_{p}+1}\left(M_{\mathrm{ph}}^{j} \hat{U}_{[i, j]}\right)\right| \varphi_{0}\right\rangle .
$$

$\left|\psi_{\mathrm{RP}}\right\rangle_{\mathrm{ph}}$ is an arbitrary RP-PEPS with open boundary condition and plaquette size $L_{p}$, with its source point at the first photonic qudit. The circuit depth is the same as that of a matter-based lattice case [cf. Eq. (1)]. The same protocol also allows us to prepare isoTNS [66], and this setup can be used to prepare photonic F-PEPS with circuit depth $O(N)$ [66].

Notice that, at the boundary of the photon source array, the photon emission process emits multiple photons, as visualized in Fig. 4(a4). In contrast to the protocol that produces RP-PEPS on a matter-based lattice, here, the overlap of gates along the horizontal direction results from acting on the ancillas. In Ref. [74], we propose a cavitytransmon setup to realize this protocol and elaborate on how to create the two-dimensional photonic cluster state and the toric code state [75].

Conclusion.-We have introduced P-PEPS and the subclass RP-PEPS, which constitute a natural generalization of sequential preparation protocols from one to higher dimensions. These states satisfy area-law entanglement by construction, combine the capacity to host long-range correlations, topologically ordered states, and a large subclass of PEPS with a simple and efficient preparation protocol. Our work helps to clarify the relation between various relevant classes of PEPS, including SGS [49], F-PEPS [50] and isoTNS [51], that we show SGS $\subset$ isoTNS $\subset$ RP-PEPS, and F-PEPS $\subset$ P-PEPS.

The family of states we introduce comes with explicit protocols that prepare them in matter-based and photonbased lattices, which makes them promising targets for near-term experimental realization. Furthermore, one can include several layers of sequential plaquettes to increase the expressivity of the ansatz.

We acknowledge funding from ERC Advanced Grant QUENOCOBA under the EU Horizon 2020 program (Grant Agreement No. 742102), and within the D-A-CH Lead-Agency Agreement through Project No. 414325145 (BEYOND C), and the European Union's Horizon 2020 research and innovation program under Grant No. 899354 (FET Open SuperQuLAN).
*These authors contributed equally.

[1] F. Verstraete, V. Murg, and J. I. Cirac, Adv. Phys. 57, 143 (2008).

[2] R. Orús, Nat. Rev. Phys. 1, 538 (2019).

[3] J. I. Cirac, D. Perez-Garcia, N. Schuch, and F. Verstraete, arXiv:2011.12127.

[4] M. Fannes, B. Nachtergaele, and R. F. Werner, Commun. Math. Phys. 144, 443 (1992).

[5] D. Perez-Garcia, F. Verstraete, M. M. Wolf, and J. I. Cirac, Quantum Inf. Comput. 7, 401 (2007).

[6] U. Schollwöck, Ann. Phys. (Amsterdam) 326, 96 (2011).

[7] M. B. Hastings, J. Stat. Mech. (2007) P08024.

[8] F. Verstraete and J. I. Cirac, Phys. Rev. B 73, 094423 (2006).

[9] F. Verstraete and J. I. Cirac, arXiv:0407066.

[10] F. Verstraete, M. M. Wolf, D. Perez-Garcia, and J. I. Cirac, Phys. Rev. Lett. 96, 220601 (2006).

[11] N. Schuch, I. Cirac, and D. Pérez-García, Ann. Phys. (Amsterdam) 325, 2153 (2010).

[12] M. A. Levin and X.-G. Wen, Phys. Rev. B 71, 045110 (2005).

[13] Z.-C. Gu, M. Levin, B. Swingle, and X.-G. Wen, Phys. Rev. B 79, 085118 (2009).

[14] O. Buerschaper, M. Aguado, and G. Vidal, Phys. Rev. B 79, 085119 (2009).

[15] C. L. Degen, F. Reinhard, and P. Cappellaro, Rev. Mod. Phys. 89, 035002 (2017).

[16] W. Dür, G. Vidal, and J. I. Cirac, Phys. Rev. A 62, 062314 (2000).

[17] D. M. Greenberger, M. A. Horne, and A. Zeilinger, in Bell's Theorem, Quantum Theory and Conceptions of the Universe (Springer, New York, 1989), pp. 69-72.

[18] H. J. Briegel and R. Raussendorf, Phys. Rev. Lett. 86, 910 (2001).

[19] M. Hein, J. Eisert, and H. J. Briegel, Phys. Rev. A 69, 062311 (2004).

[20] A. Russo, E. Barnes, and S. E. Economou, New J. Phys. 21, 055002 (2019).

[21] K. Azuma, K. Tamaki, and H. K. Lo, Nat. Commun. 6, 6787 (2015).

[22] C. Kruszynska and B. Kraus, Phys. Rev. A 79, 052304 (2009).

[23] M. Rossi, M. Huber, D. Bruß, and C. Macchiavello, New J. Phys. 15, 113022 (2013).

[24] C. Schön, E. Solano, F. Verstraete, J. I. Cirac, and M. M. Wolf, Phys. Rev. Lett. 95, 110503 (2005).

[25] C. Schön, K. Hammerer, M. M. Wolf, J. I. Cirac, and E. Solano, Phys. Rev. A 75, 032311 (2007).

[26] A. Smith, B. Jobst, A. G. Green, and F. Pollmann, arXiv: 1910.05351.

[27] S.-H. Lin, R. Dilip, A. G. Green, A. Smith, and F. Pollmann, PRX Quantum 2, 010342 (2021).

[28] K. M. Gheri, C. Saavedra, P. Törmä, J. I. Cirac, and P. Zoller, Phys. Rev. A 58, R2627 (1998).

[29] C. Saavedra, K. M. Gheri, P. Törmä, J. I. Cirac, and P. Zoller, Phys. Rev. A 61, 062311 (2000).

[30] N. H. Lindner and T. Rudolph, Phys. Rev. Lett. 103, 113602 (2009).

[31] I. Schwartz, D. Cogan, E. R. Schmidgall, Y. Don, L. Gantz, O. Kenneth, N. H. Lindner, and D. Gershoni, Science 354, 434 (2016). 
[32] K. Tiurev, M. H. Appel, P. L. Mirambell, M. B. Lauritzen, A. Tiranov, P. Lodahl, and A.S. Sørensen, arXiv:2007 .09295 .

[33] C. Eichler, J. Mlynek, J. Butscher, P. Kurpiers, K. Hammerer, T. J. Osborne, and A. Wallraff, Phys. Rev. X 5, 041044 (2015).

[34] J.-C. Besse, K. Reuer, M. C. Collodo, A. Wulff, L. Wernli, A. Copetudo, D. Malz, P. Magnard, A. Akin, M. Gabureac, G. J. Norris, J. I. Cirac, A. Wallraff, and C. Eichler, Nat. Commun. 11, 4877 (2020).

[35] Z.-Y. Wei, D. Malz, A. González-Tudela, and J. I. Cirac, Phys. Rev. Research 3, 023021 (2021).

[36] S. C. Wein, J. C. Loredo, M. Maffei, P. Hilaire, A. Harouri, N. Somaschi, A. Lemaître, I. Sagnes, L. Lanco, O. Krebs, A. Auffèves, C. Simon, P. Senellart, and C. Antón-Solanas, arXiv:2106.02049.

[37] L. Lamata, J. León, D. Pérez-García, D. Salgado, and E. Solano, Phys. Rev. Lett. 101, 180506 (2008).

[38] M. Cramer, M. B. Plenio, S. T. Flammia, R. Somma, D. Gross, S. D. Bartlett, O. Landon-Cardinal, D. Poulin, and Y. K. Liu, Nat. Commun. 1, 149 (2010).

[39] H. Saberi, Phys. Rev. A 84, 032323 (2011).

[40] Y. Delgado, L. Lamata, J. León, D. Salgado, and E. Solano, Phys. Rev. Lett. 98, 150502 (2007).

[41] T. J. Osborne, J. Eisert, and F. Verstraete, Phys. Rev. Lett. 105, 260401 (2010).

[42] W. Huggins, P. Patil, B. Mitchell, K. Birgitta Whaley, and E. Miles Stoudenmire, Quantum Sci. Technol. 4, 024001 (2019).

[43] M. Foss-Feig, D. Hayes, J. M. Dreiling, C. Figgatt, J. P. Gaebler, S. A. Moses, J. M. Pino, and A. C. Potter, Phys. Rev. Research 3, 033002 (2021).

[44] F. Barratt, J. Dborin, M. Bal, V. Stojevic, F. Pollmann, and A. G. Green, npj Quantum Inf. 7, 79 (2021).

[45] S. J. Ran, Phys. Rev. A 101, 032310 (2020).

[46] N. Schuch, M. M. Wolf, F. Verstraete, and J. I. Cirac, Phys. Rev. Lett. 98, 140506 (2007).

[47] K. J. Satzinger, Y. Liu, A. Smith, C. Knapp, M. Newman, C. Jones, Z. Chen, C. Quintana, X. Mi, A. Dunsworth, and Others, arXiv:2104.01180.

[48] Y.-J. Liu, K. Shtengel, A. Smith, and F. Pollmann, arXiv: 2110.02020.

[49] M. C. Bañuls, D. Pérez-García, M. M. Wolf, F. Verstraete, and J. I. Cirac, Phys. Rev. A 77, 052306 (2008).

[50] H. Pichler, S. Choi, P. Zoller, and M. D. Lukin, Proc. Natl. Acad. Sci. U.S.A. 114, 11362 (2017).

[51] M. P. Zaletel and F. Pollmann, Phys. Rev. Lett. 124, 037201 (2020).
[52] T. Soejima, K. Siva, N. Bultinck, S. Chatterjee, F. Pollmann, and M. P. Zaletel, Phys. Rev. B 101, 085117 (2020).

[53] S. E. Economou, N. Lindner, and T. Rudolph, Phys. Rev. Lett. 105, 093601 (2010).

[54] M. Gimeno-Segovia, T. Rudolph, and S. E. Economou, Phys. Rev. Lett. 123, 070501 (2019).

[55] R. Bekenstein, I. Pikovski, H. Pichler, E. Shahmoon, S. F. Yelin, and M. D. Lukin, Nat. Phys. 16, 676 (2020).

[56] I. Dhand, M. Engelkemeier, L. Sansoni, S. Barkhofen, C. Silberhorn, and M. B. Plenio, Phys. Rev. Lett. 120, 130501 (2018).

[57] S. Xu and S. Fan, APL Photonics 3, 116102 (2018).

[58] K. Wan, S. Choi, I. H. Kim, N. Shutty, and P. Hayden, arXiv:2011.08213 [Phys. Rev. X (to be published)].

[59] Y. Zhan and S. Sun, Phys. Rev. Lett. 125, 223601 (2020).

[60] Y. Shi and E. Waks, Phys. Rev. A 104, 013703 (2021).

[61] S. Bartolucci, P. Birchall, H. Bombin, H. Cable, C. Dawson, M. Gimeno-Segovia, E. Johnston, K. Kieling, N. Nickerson, M. Pant, F. Pastawski, T. Rudolph, and C. Sparrow, arXiv: 2101.09310.

[62] H. Bombin, I. H. Kim, D. Litinski, N. Nickerson, M. Pant, F. Pastawski, S. Roberts, and T. Rudolph, arXiv:2103.08612.

[63] A. Blais, A. L. Grimsmo, S. M. Girvin, and A. Wallraff, Rev. Mod. Phys. 93, 025005 (2021).

[64] C. D. Bruzewicz, J. Chiaverini, R. McConnell, and J. M. Sage, Appl. Phys. Rev. 6, 021314 (2019).

[65] M. Saffman, J. Phys. B 49, 202001 (2016).

[66] See Supplemental Material at http://link.aps.org/ supplemental/10.1103/PhysRevLett.128.010607 for more details, which includes Refs. [67,68].

[67] M. S. J. Tepaske and D. J. Luitz, Phys. Rev. Research 3, 023236 (2021).

[68] R. Raussendorf, J. Harrington, and K. Goyal, Ann. Phys. (Amsterdam) 321, 2242 (2006).

[69] S. Gopalakrishnan and A. Lamacraft, Phys. Rev. B 100, 064309 (2019).

[70] A. Nahum, J. Ruhman, S. Vijay, and J. Haah, Phys. Rev. X 7, 031016 (2017).

[71] A. Nahum, S. Vijay, and J. Haah, Phys. Rev. X 8, 021014 (2018).

[72] C. W. Von Keyserlingk, T. Rakovszky, F. Pollmann, and S. L. Sondhi, Phys. Rev. X 8, 021013 (2018).

[73] R. Haghshenas, M. J. O’Rourke, and G. K. L. Chan, Phys. Rev. B 100, 054404 (2019).

[74] Z.-Y. Wei, J. I. Cirac, and D. Malz, arXiv:2109.06781.

[75] Note that, for the cluster state generation, there are specific schemes $[30,53]$ that do not need ancillas and SWAP gates. Thus, these schemes require less resources than the present scheme. 